\title{
WINDING NUMBERS AND FULL EXTENDIBILITY IN HOLOMORPHIC MOTIONS
}

\author{
YUNPING JIANG
}

\begin{abstract}
We construct an example of a holomorphic motion of a five-point subset of the Riemann sphere over an annulus such that it satisfies the zero winding number condition but is not fully extendable.
\end{abstract}

\section{INTRODUCTION}

Suppose $\mathbb{C}$ is the complex plane and $\widehat{\mathbb{C}}=\mathbb{C} \cup\{\infty\}$ is the Riemann sphere. Let

$$
\Delta=\{c \in \mathbb{C}|| c \mid<1\}
$$

be the open unit disk. Suppose $X$ is a connected complex manifold with a basepoint $t_{0}$. Let $E \subset \widehat{\mathbb{C}}$ be a subset. A map $\phi(t, z): X \times E \rightarrow \widehat{\mathbb{C}}$ is called a holomorphic motion of $E$ over $X$ if

i) $\phi\left(t_{0}, z\right)=z$ for all $z \in E$;

ii) for any fixed $t \in X, \phi_{t}(\cdot)=\phi(t, \cdot): E \rightarrow \widehat{\mathbb{C}}$ is injective;

iii) for any fixed $z \in E, \phi^{z}(\cdot)=\phi(\cdot, z): X \rightarrow \widehat{\mathbb{C}}$ is holomorphic.

By pre- and postcompositing Möbius transformations, without loss of generality, we can always assume that $E$ contains 0,1 , and $\infty$ and $\phi$ is normalized, that is, $\phi(t, z)=z$ for $z=0,1$, and $\infty$ and all $t \in X$. Henceforth, all holomorphic motions in this paper are normalized.

We say a holomorphic motion $\phi$ of $E$ over $X$ is fully extendable if there is a holomorphic motion $\psi$ of $\widehat{\mathbb{C}}$ over $X$ such that the restriction

$$
\psi \mid X \times E=\phi .
$$

We call $\psi$ a full extension of $\phi$.

Slodkowski's Theorem (see [8, 13]) says that any holomorphic motion of $E$ over $\Delta$ is fully extendable. There is an example of a holomorphic motion of $E$ over a nonsimply connected one-dimensional hyperbolic complex manifold $X$, which is not fully extendable (see, for example, [7]). This leads us to consider the following zero winding number condition in holomorphic motions of $E$ over a one-dimensional hyperbolic complex manifold $X$ as follows.

Received by the editors February 6, 2020.

2010 Mathematics Subject Classification. Primary 32G15; Secondary 30C99, 37F30.

Key words and phrases. Holomorphic motions, fully extendable, connected one-dimensional hyperbolic complex manifold, the zero winding number condition.

This material is based upon work supported by the National Science Foundation. It is also partially supported by a collaboration grant from the Simons Foundation (grant number 523341) and PSC-CUNY awards. 
Suppose $\alpha$ is a closed curve in $\mathbb{C}$ not containing 0 . Then we can consider its winding number

$$
\eta=\eta(\alpha)=\frac{1}{2 \pi} \oint_{\alpha} d \arg \alpha .
$$

The winding number is an integer and $2 \pi \eta$ is just the variation of argument on $\alpha$. Given a holomorphic motion $\phi$ of $E$ over a one-dimensional hyperbolic complex manifold $X$ and given a simple closed curve $\beta$ in $X$ and a pair of points $z_{1} \neq z_{2} \in E$, we have the winding number $\eta(\alpha)$ of the closed curve

$$
\alpha=\alpha\left(\beta, z_{1}, z_{2}\right)=\phi\left(\beta, z_{1}\right)-\phi\left(\beta, z_{2}\right) \subset \widehat{\mathbb{C}} .
$$

We say $\phi$ satisfies the zero winding number condition if $\eta(\alpha)=0$ for every simple closed curve $\beta$ in $X$ and every pair of points $z_{1} \neq z_{2} \in E$.

Chirka gave a wonderful new proof of Slodkowski's Theorem (see $[5,8]$ ). Further, he claimed in [5] that the zero winding number condition is a necessary and sufficient condition for a fully extendable holomorphic motion of $E$ over any connected onedimensional hyperbolic complex manifold $X$. Since then, the following problem has arisen in the study of holomorphic motions: Is the zero winding number condition a sufficient condition for a fully extendable holomorphic motion of $E$ over a connected one-dimensional hyperbolic complex manifold $X$ ? The purpose of this paper is to show that the zero winding number condition is not sufficient.

\section{THE MAIN RESULT}

Consider two arcs

$$
T^{+}=\{t=x+y i \in \mathbb{C}|| t+i / 2 \mid=\sqrt{5} / 2, y \geq 0\}
$$

and

$$
T^{-}=\left\{\frac{1}{t} \mid t \in T^{+}\right\} .
$$

Then $T=T^{+} \cup T^{-}$is a simple closed curve such that $-1,1 \in T$ (see Figure 1 ). It cuts $\mathbb{C}$ into two domains, one is bounded and simply connected domain $D$ and the other is unbounded and connected domain $\widetilde{D}$. Then we have $0,-i \in D$, and $i \in \widetilde{D}$ (see Figure 1). Consider an annulus

$$
X=\{t \in \mathbb{C} \mid \operatorname{dist}(t, T)<\epsilon\}, \quad \epsilon>0,
$$

where $\operatorname{dist}(t, T)$ means the distance between $t$ and $T$. Choose $\epsilon$ small enough such that $-2,0,1 / 2,1 / 3, i,-i \notin X$. Take $t_{0}=1$ as the basepoint of $X$ (see Figure 1 ).

Let

$$
S^{+}=\left\{-\frac{1}{t} \mid t \in T^{+}\right\} \quad \text { and } \quad S^{-}=\left\{-\frac{1}{t} \mid t \in T^{-}\right\}
$$

and $S=S^{+} \cup S^{-}$. It is again a simple closed curve such that $-1,1 \in S$, and let

$$
Y=\{t \in \mathbb{C} \mid \operatorname{dist}(t, S)<\epsilon\}=\left\{-\frac{1}{t} \mid t \in X\right\}
$$

be another annulus (see Figure 1).

Let $E=\{0,1,2,4, \infty\} \subset \widehat{\mathbb{C}}$ be a five-point subset in $\widehat{\mathbb{C}}$. Define

$$
\phi(t, z)= \begin{cases}z & \text { if } z=0,1, \infty \text { and } t \in X \\ -\frac{1}{t}+3 & \text { if } z=2 \text { and } t \in X \\ t+3 & \text { if } z=4 \text { and } t \in X\end{cases}
$$



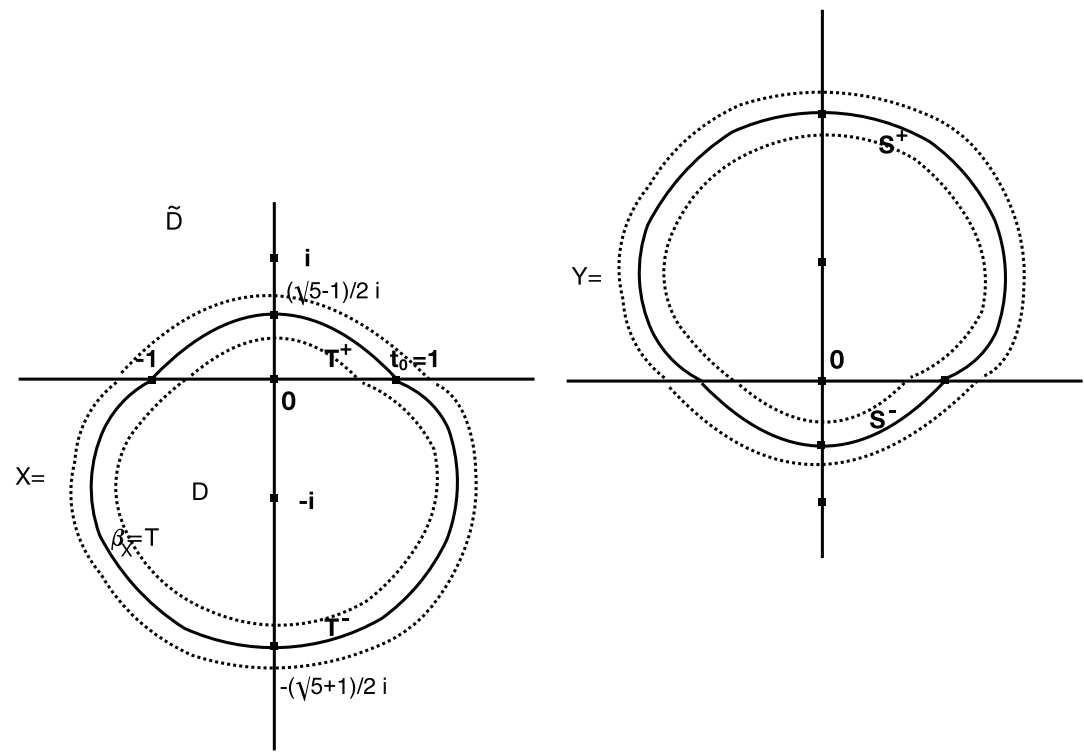

FiguRE 1. An annulus with a basepoint as a parameter space

We check that it is a holomorphic motion as follows.

(i) $\phi\left(t_{0}, z\right)=z$ for all $z \in E$.

(ii) First $-1 / t+3$ and $t+3$ will not take $0,1, \infty$ for any $t \in X$, and secondly, since $X$ does not contain $i$ and $-i,-1 / t \neq t$ for for any $t \in X$. Thus for any fixed $t \in X, \phi_{t}(\cdot)=\phi(t, \cdot): E \rightarrow \widehat{\mathbb{C}}$ is injective.

(iii) Since $X$ does not contain $0,-(1 / t)+3$ is holomorphic on $X$. Therefore, for any fixed $z \in E, \phi^{z}(\cdot)=\phi(\cdot, z): X \rightarrow \widehat{\mathbb{C}}$ is holomorphic.

Thus $\phi(t, z)$ is a holomorphic motion of $E$ over $X$. The main statement of this paper is as presented in the following theorem.

Theorem 1 (Main Theorem). The holomorphic motion $\phi$ of $E$ over $X$ defined in (2) satisfies the zero winding number condition but is not fully extendable.

Proof. We first check the zero winding number condition for $\phi$ in (2). The set $E$ contains only five points $0,1,2,4$, and $\infty$, and $X$ has only one nontrivial simple closed curve $\beta_{X}=T$ in the sense of homotopy. Thus we need only to consider the winding number $\eta(\alpha)$ of

$$
\alpha=\alpha\left(\beta_{X}, z_{1}, z_{2}\right)=\phi\left(\beta_{X}, z_{1}\right)-\phi\left(\beta_{X}, z_{2}\right)
$$

for any pair of points $z_{1} \neq z_{2} \in E$.

When $z_{1}, z_{2}=0,1, \infty$, it is clear that $\eta(\alpha)=0$. For $z_{1}=4$ and $z_{2}=0,1, \infty$, then we have that $\alpha=\beta_{X}+3, \beta_{X}+2,1 /\left(\beta_{X}+3\right)$ (for $z_{2}=\infty$, we use the coordinate $w=1 / z)$. Since 0 is in the unbounded component of $\mathbb{C} \backslash \alpha$ in these cases, we have $\eta(\alpha)=0$. For $z_{1}=2$ and $z_{2}=0,1, \infty$, then we have that $\alpha=-\left(1 / \beta_{X}\right)+3$, $-\left(1 / \beta_{X}\right)+2,-\beta_{X}+3$ (for $z_{2}=\infty$, we use the coordinate $w=1 / z$ ). In these cases, since 0 is in the unbounded component of $\mathbb{C} \backslash \alpha$, we have $\eta(\alpha)=0$. Symmetrically, when $z_{1}=0,1, \infty$ and $z_{2}=2,4$, we also have $\eta(\alpha)=0$. 
When $z_{1}=4$ and $z_{2}=2$,

$$
\alpha=\phi\left(\beta_{X}, 4\right)-\phi\left(\beta_{X}, 2\right)=\beta_{X}+\frac{1}{\beta_{X}} .
$$

It is the image of the simple closed curve $\beta_{X}$ under the map $f(z)=t+1 / t$. The map $f(t)$ is a meromorphic function on the simply connected domain $D$ enclosed by $\beta_{X}$ and is nonzero and continuous on $\beta_{X}$ (see Figure 1). It has one zero (counted by multiplicity) at $-i$ and one pole (counted by multiplicity) at 0 inside $D$. From the argument principle in complex analysis, the winding number

$$
\eta(\alpha)=\frac{1}{2 \pi i} \oint_{\beta_{X}} \frac{f^{\prime}(z)}{f(z)} d z=1-1=0 .
$$

Symmetrically, when $z_{1}=2$ and $z_{2}=4$, we also have $\eta(\alpha)=0$. We completed the proof that $\phi$ satisfies the zero winding number condition.

To prove that $\phi$ is not fully extendable, we need to introduce the isotopy rel $E$ among all homeomorphisms $h$ of $\widehat{\mathbb{C}}$. Two homeomorphisms $h_{0}$ and $h_{1}$ of $\widehat{\mathbb{C}}$ are said to be isotopic rel $E$, denoted as $h_{1} \sim_{E} h_{0}$, if there is a continuous map

$$
H(s, z):[0,1] \times \widehat{\mathbb{C}} \rightarrow \widehat{\mathbb{C}}
$$

such that

(a) $H(1, z)=h_{0}^{-1} \circ h_{1}(z)$ and $H(0, z)=z$ for all $z \in \widehat{\mathbb{C}}$;

(b) $h_{s}(\cdot)=H(s, \cdot)$ is a homeomorphism of $\widehat{\mathbb{C}}$ for every $s \in[0,1]$;

(c) $H(s, z)=z$ for all $z \in E$ and $s \in[0,1]$.

We use $[h]_{E}$ to denote the isotopic class of $h$ rel $E$. We call $h$ isotopic trivial rel $E$ if $[h]_{E}=[I d]_{E}$; otherwise, we call $h$ isotopic nontrivial rel $E$.

Consider the annulus $A=Y+3$ and the simple closed curve $\beta_{Y}=S+3$ which is homotopic to the core curve of $A$. Let $\tau$ be the Dehn twist on $A$ along the curve $\beta_{Y}$ by rotating $2 \pi$ clockwise.

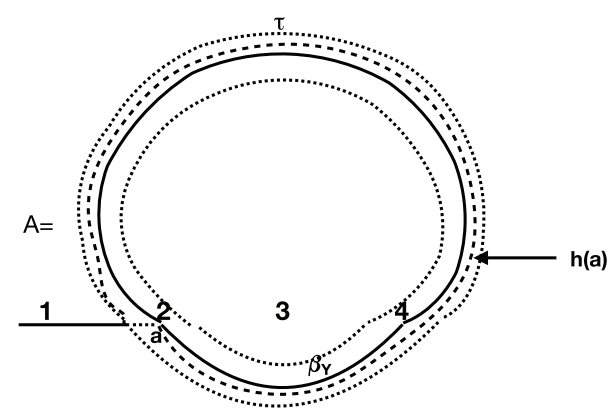

Figure 2. A Dehn twist on the annulus along its core curve

Lemma 1. Suppose $H$ is a homeomorphism of $\widehat{\mathbb{C}}$ fixing every point in $E$ such that $H \mid A$ is $\tau$ in the sense of homotopy (See Figure 2). Then $H$ is isotopic nontrivial rel E.

Proof. Consider the Riemann surface $S=\widehat{\mathbb{C}} \backslash E$. Given two simple closed curves $A$ and $B$ in $S$, the intersection number $I(A, B)$ is by the definition the minimum number among \# $\left(A^{\prime} \cap B^{\prime}\right)$ for all simple closed curves $A^{\prime}$ homotopic to $A$ in $S$ and all simple closed curves $B^{\prime}$ homotopic to $B$ in $S$. 
Suppose $H$ is a homeomorphism of $\widehat{\mathbb{C}}$ fixing every point in $E$. Suppose $A$ is a simple closed curve in $S$. Let $B=H(A)$ and consider $I(A, B)$. Then saying that $H$ is isotopic trivial rel $E$ implies that $I(A, B)=0$ for every simple closed curve $A$ in $S$ (refer to [4]). We will use this fact in the hyperbolic geometry to show that $H$ in the lemma is isotopic nontrivial rel $E$.

Let

$$
A=\left\{z \in \mathbb{C}|| z-\frac{3}{2} \mid=1\right\} .
$$

Then 1 and 2 are in the bounded component of $\mathbb{C} \backslash A$ and 0 and 4 are in the unbounded component of $\mathbb{C} \backslash A$. It is a simple closed curve in $S=\mathbb{C} \backslash E$. Since $H$ restricted to $Y$ is a Dehn twist along $\beta_{Y}$ by rotating $2 \pi$ closewise, $B=h(A)$ is also a simple closed curve in $S$ such that 1 and 2 are in the bounded component of $\mathbb{C} \backslash B$ and 0 and 4 are in the unbounded component of $\mathbb{C} \backslash B$. (See Figure 3). However, one can check that the intersection number $I(A, B)=4$. This implies that $H$ must be isotopic nontrivial rel $E$.

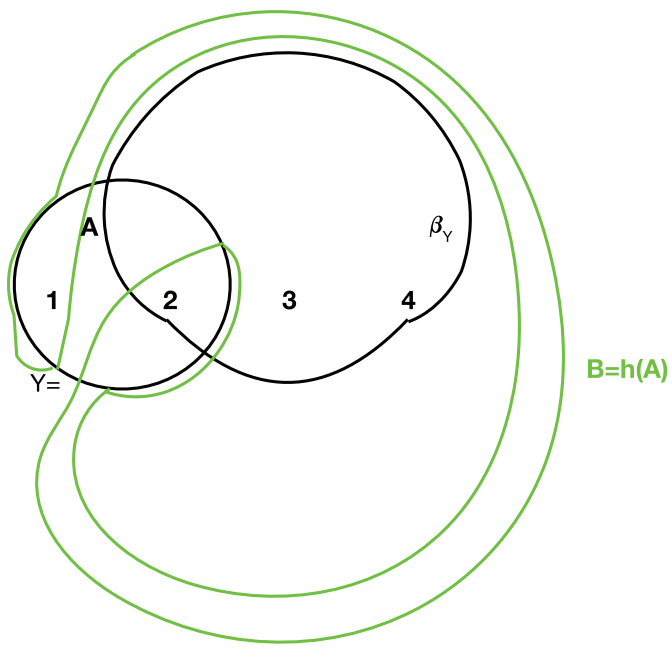

Figure 3. The image of a simple closed curve under the Dehn twist

Let $M(\mathbb{C})$ be the space of all $L^{\infty}$ complex functions $\mu$ on $\mathbb{C}$ with norms $\|\mu\|_{\infty}<1$, which is a simply connected complex Banach manifold. For every $\mu \in M(\mathbb{C})$, consider the Beltrami equation

$$
w_{\bar{z}}=\mu w_{z} .
$$

A solution $w$ of (3) is called a quasiconformal homeomorphism of $\widehat{\mathbb{C}}$. If $w(0)=0$, $w(1)=1$, and $w(\infty)=\infty$, it is called a normalized solution. Here $\mu$ is called the Beltrami coefficient. The measurable Riemann mapping theorem says that for every $\mu \in M(\mathbb{C})$, the Beltrami equation (3) has a unique normalized solution, denoted as $w^{\mu}$. Moreover, $w^{\mu}$ depends on $\mu$ holomorphically (refer to [1,8]). Then we can define a normalized holomorphic motion

$$
\Phi(\mu, z)=w^{\mu}(z): M(\mathbb{C}) \times \widehat{\mathbb{C}} \rightarrow \widehat{\mathbb{C}} .
$$

To continue our proof, we consider the fundamental group $\pi_{1}(X)=\mathbb{Z}$, the integer group, with the homotopic class $\left[\beta_{X}\right]=1$. Let $\pi: \Delta \rightarrow X$ be the holomorphic 
universal cover such that $\pi(0)=t_{0}$. Let $\Gamma$ be the corresponding group of deck transformations. Let $\gamma$ be the deck transformation (a Möbius transformation on $\Delta$ ) corresponding to $\left[\beta_{X}\right]$; then $\Gamma=\langle\gamma\rangle$. Let $\delta$ be the geodesic connecting 0 and $\gamma(0)$ in $\Delta$; then $[\pi(\delta)]=\left[\beta_{X}\right]=1$. (See Figure 4 ).

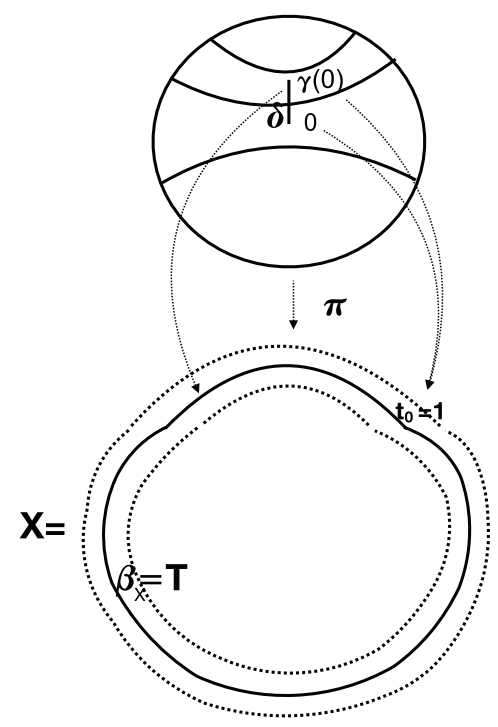

Figure 4. The universal cover of an annulus and its fundamental group

Pull back $\phi$ by $\pi$. We have a holomorphic motion

$$
\widetilde{\phi}(c, z)=\phi(\pi(c), z): \Delta \times E \rightarrow \widehat{\mathbb{C}},
$$

which is fully extendable as we have mentioned in the introduction. Let $\widetilde{\psi}(c, z)$ : $\Delta \times \widehat{\mathbb{C}} \rightarrow \widehat{\mathbb{C}}$ be a full extension of $\widetilde{\phi}$. For each $c \in \Delta, \widetilde{\psi_{c}}: \widehat{\mathbb{C}} \rightarrow \widehat{\mathbb{C}}$ is a quasiconformal homeomorphism (refer to $[2,8,11,12]$ ). Let $\mu(c)$ be the Beltrami coefficient of $\widetilde{\psi}_{c}$. Then

$$
F(c)=\mu(c): \Delta \rightarrow M(\mathbb{C}), F(0)=0
$$

defines a holomorphic map (refer to $2,8,11,12$ ) such that

$$
\widetilde{\psi}(c, z)=w^{F(c)}(z): \Delta \times \widehat{\mathbb{C}} \rightarrow \widehat{\mathbb{C}}
$$

is a full extension of $\widetilde{\phi}(c, z): \Delta \times E \rightarrow \widehat{\mathbb{C}}$.

When $c$ moves from 0 to $\gamma(0)$ along $\delta$ in $\Delta, t=\pi(c)$ moves the point 1 back to 1 counterclockwise on $\beta_{X}$ in $X$ (more precisely, on the unique nontrivial closed geodesic homotopic to $\beta_{X}$ in $X$ ) and

$$
\widetilde{\psi}(c, 2)=\widetilde{\phi}(c, 2)=-\frac{1}{t}+3
$$

moves the point 2 back to the point 2 clockwise on $\beta_{Y}$ in $Y$. Thus we have the following corollary.

Corollary 1. The homeomorphism

$$
H(\cdot)=\widetilde{\psi}(\gamma(0), \cdot)=w^{F(\gamma(0))}(\cdot)
$$


of $\widehat{\mathbb{C}}$ fixes every point in $E$ and the restriction $H \mid A$ is the Dehn twist $\tau$ in the sense of homotopy. Thus from Lemma 1, $H$ is isotopic nontrivial rel $E$.

We need the next lemma to make sure that the choice of $F$ will not change the isotopic nontrivial property for $H$.

Lemma 2. The isotopic class $[H]_{E}$ does not depend on the choice of any continuous map $F$ in (4) satisfying (5).

Proof. Let $F_{1}$ and $F_{2}$ be two maps in (4) satisfying (5). Parametrize $\delta$ as a path $\delta(s):[0,1] \rightarrow \Delta$ from $\gamma(0)$ to 0 . Let

$$
H(s, z)=\left(w^{F_{1}(\gamma(0))}\right)^{-1} \circ w^{F_{1}(\delta(s))} \circ\left(w^{F_{2}(\delta(s))}\right)^{-1} \circ w^{F_{2}(\gamma(0))}(z):[0,1] \times \widehat{\mathbb{C}} \rightarrow \widehat{\mathbb{C}} .
$$

Then we have that for $s=0, \delta(0)=\gamma(0), H(0, z)=z$ for all $z \in \widehat{\mathbb{C}}$. For $s=1$, $\delta(1)=0$, since $F_{1}(0)=F_{2}(0)=0, H(1, z)=\left(w^{F_{1}(\gamma(0))}\right)^{-1} \circ w^{F_{2}}(\gamma(0))$. For $z \in E$ and $s \in[0,1], w^{F_{1}(\delta(s))}(z)=w^{F_{2}(\delta(s))}(z)=\widetilde{\phi}(\delta(s), z)$, we have that $H(s, z)=z$. We see that $H(\cdot, \cdot)$ gives an isotopy between $w^{F_{1}(\gamma(0))}$ and $w^{F_{2}(\gamma(0))}$ rel $E$, that is, $w^{F_{1}(\gamma(0))} \sim_{E} w^{F_{2}(\gamma(0))}$. This completes the proof.

Now we complete our proof of Theorem 1 by using the contradiction. That is, we will show that if $\phi$ is fully extendable, then $H(\cdot)=\widetilde{\psi}(\gamma(0), \cdot)$ is isotopic trivial rel $E$.

Suppose $\phi$ is fully extendable and suppose $\psi(t, z): X \times \widehat{\mathbb{C}} \rightarrow \widehat{\mathbb{C}}$ is a full extension of $\phi$. For each $t \in X, \psi(t, \cdot): \widehat{\mathbb{C}} \rightarrow \widehat{\mathbb{C}}$ is a quasiconformal homeomorphism (refer to $[2,8,11,12])$. Let $\mu(t)$ be the Beltrami coefficient of $\psi(t, \cdot)$. Then

$$
F_{X}(t)=\mu(t): X \rightarrow M(\mathbb{C}), F_{X}\left(t_{0}\right)=0
$$

defines a holomorphic map (refer to $[2,8,11,12$ ) such that

$$
\psi(t, z)=w^{F_{X}(t)}(z), t \in X .
$$

We can now take $F=F_{X} \circ \pi: \Delta \rightarrow M(\mathbb{C})$ as a map in (4) satisfying (5) such that

$$
H(\cdot)=\widetilde{\psi}(\gamma(0), \cdot)=w^{F(\gamma(0))}(\cdot) .
$$

This implies that

$$
[H]_{E}=\left[w^{F(\gamma(0))}\right]_{E}=\left[w^{F_{X}(\pi(\gamma(0)))}\right]_{E}=\left[w^{F_{X}\left(t_{0}\right)}\right]_{E}=[I d]_{E} .
$$

This says that $H$ is isotopic trivial rel $E$. It contradicts Corollary 1, The contradiction says that $\phi$ cannot be fully extendable. This completes the proof of Theorem 1.

\section{AdDED REMARK}

I would like to thank the referee who suggested that I give a connection of the result in this paper with pure braid groups as defined in John Hubbard's book 9, $\S 5.2]$. Actually, I implicitly used the pure braid of five threads (see Figure 5) in the proof of the main theorem (Theorem 11). A well-known result in the theory of braids (see, e.g., 6, Theorem 1.33]) says that such a braid corresponds to a nontrivial mapping class group of the configuration space. Using pure braids, we defined the monodromy associated with a holomorphic motion in [3]. We have the following theorem in [3, Proposition 2.13] (see [9, §5.2] for the version by using pure braids). Let $V$ be a complex Banach manifold, and let $E$ be a closed subset in $\widehat{\mathbb{C}}$ containing $0,1, \infty$. 


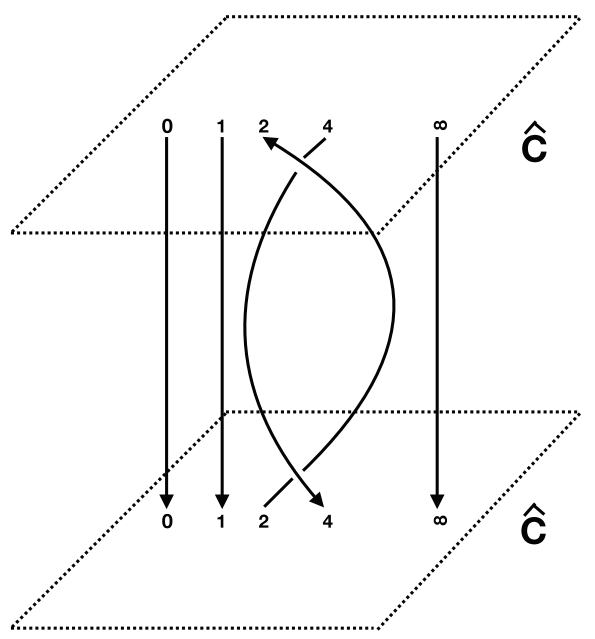

FiguRE 5. The pure braid of five threads given in (2)

Theorem 2. If $\phi: V \times E \rightarrow \widehat{\mathbb{C}}$ is a fully extendable holomorphic motion, then $\phi$ restricted to $V \times E^{\prime}$ has trivial monodromy, where $\{0,1, \infty\} \subset E^{\prime}$ is any finite subset of $E$.

Furthermore, we have the following theorem in [10, Theorem D]. Let $X$ be a hyperbolic Riemann surface, and let $E$ be a closed subset in $\widehat{\mathbb{C}}$ containing $0,1, \infty$.

Theorem 3. Let $\phi: X \times E \rightarrow \widehat{\mathbb{C}}$ be a holomorphic motion such that $\phi$ restricted to $X \times E^{\prime}$ has trivial monodromy, where $\{0,1, \infty\} \subset E^{\prime}$ is any finite subset of $E$. Then $\phi$ is fully extendable.

Thus the triviality of the monodromy associated with a holomorphic motion $\phi: X \times E \rightarrow \widehat{\mathbb{C}}$ gives a complete topological characterization of a fully extendable holomorphic motion. The pure braid of five threads given in (2) is illustrated in Figure 5. Thus, the monodromy associated with the holomorphic motion given in (2) is nontrivial. This gives another explanation as to why the holomorphic motion defined in (2) is not fully extendable.

\section{REFERENCES}

[1] Lars V. Ahlfors, Lectures on quasiconformal mappings, 2nd ed., University Lecture Series, vol. 38, American Mathematical Society, Providence, RI, 2006. With supplemental chapters by C. J. Earle, I. Kra, M. Shishikura and J. H. Hubbard. MR2241787

[2] Lipman Bers and H. L. Royden, Holomorphic families of injections, Acta Math. 157 (1986), no. 3-4, 259-286, DOI 10.1007/BF02392595. MR857675

[3] Michael Beck, Yunping Jiang, Sudeb Mitra, and Hiroshige Shiga, Extending holomorphic motions and monodromy, Ann. Acad. Sci. Fenn. Math. 37 (2012), no. 1, 53-67, DOI 10.5186/aasfm.2012.3713. MR2920423

[4] Andrew J. Casson and Steven A. Bleiler, Automorphisms of surfaces after Nielsen and Thurston, London Mathematical Society Student Texts, vol. 9, Cambridge University Press, Cambridge, 1988. MR964685

[5] E. M. Chirka, On the propagation of holomorphic motions (Russian), Dokl. Akad. Nauk 397 (2004), no. 1, 37-40. MR2117461 
[6] Christian Kassel and Vladimir Turaev, Braid groups, Graduate Texts in Mathematics, vol. 247, Springer, New York, 2008. With the graphical assistance of Olivier Dodane. MR.2435235

[7] Clifford J. Earle, Some maximal holomorphic motions, Lipa's legacy (New York, 1995), Contemp. Math., vol. 211, Amer. Math. Soc., Providence, RI, 1997, pp. 183-192, DOI 10.1090/conm/211/02820. MR 1476987

[8] Frederick P. Gardiner, Yunping Jiang, and Zhe Wang, Holomorphic motions and related topics, Geometry of Riemann surfaces, London Math. Soc. Lecture Note Ser., vol. 368, Cambridge Univ. Press, Cambridge, 2010, pp. 156-193. MR2665009

[9] J. Hubbard, Teichmüller Theory, Matrix Editions, Ithaca, NY 14850.

[10] Yunping Jiang and Sudeb Mitra, Monodromy, liftings of holomorphic maps, and extensions of holomorphic motions, Conform. Geom. Dyn. 22 (2018), 333-344, DOI 10.1090/ecgd/329. MR3886152

[11] Yunping Jiang and Sudeb Mitra, Douady-Earle section, holomorphic motions, and some applications, Quasiconformal mappings, Riemann surfaces, and Teichmüller spaces, Contemp. Math., vol. 575, Amer. Math. Soc., Providence, RI, 2012, pp. 219-251, DOI 10.1090/conm/575/11400. MR2933902

[12] Sudeb Mitra, Extensions of holomorphic motions, Israel J. Math. 159 (2007), 277-288, DOI 10.1007/s11856-007-0047-1. MR.2342482

[13] Zbigniew Slodkowski, Holomorphic motions and polynomial hulls, Proc. Amer. Math. Soc. 111 (1991), no. 2, 347-355, DOI 10.2307/2048323. MR.1037218

Department of Mathematics, Queens College of the City University of New York, Flushing, New York 11367-1597; Department of Mathematics, Graduate Center of the City University of New York, New York, New York 10016

Email address: yunping.jiang@qc.cuny.edu 